\title{
Evaluation of Microfluidics-FISH method in prenatal diagnosis
}

\author{
Aleksandra Pietrzyk ${ }^{1,2}$, Małgorzata Ryłów², Marta Bryśkiewicz², Ewa Studniak², \\ Krzysztof Piotrowski ${ }^{3}$, Stanisław Zajączek ${ }^{1,2}$, Jacek Gronwald ${ }^{1,2}$ \\ ${ }^{1}$ Department of Genetics and Pathology, Pomeranian Medical University, Szczecin, Poland \\ ${ }^{2}$ Cytogenetic Unit, Department of Laboratory Diagnostics, Pomeranian Medical University, Szczecin, Poland \\ ${ }^{3}$ Department of Genetics and Pathology, Pomeranian Medical University, Szczecin, Poland
}

\begin{abstract}
Objectives: Classical cytogenetic analysis remains a gold standard in invasive prenatal diagnosis. Recently, Microfluidics-FISH, a novel method based on FISH, has been introduced. This integral approach allows to obtain result for common aneuploidies within the same day from a much smaller sample of the amniotic fluid. In this study we compare effectiveness of Microfluidics-FISH to classical karyotype and Rapid FISH.

Material and methods: 52 samples of amniotic fluid were drawn from the pregnant women due to common indications. Cell cultures have been set up for classical cytogenetic analysis as well as amniotic cells have been loaded into the microchip of Microfluidics-FISH as well standard procedure of Rapid FISH was performed for evaluation of trisomy 21, 13, 18 chromosome and sex chromosomes numeric aberrations.

Results: 9 samples out of 52 showed chromosomal aberrations in both FISH methods what was consistent with karyotyping. One case of small supernumerary marker chromosome was detected only in the classical cytogenetic analysis. For the majority of cases turnaround time was shortest for Microfluidics-FISH and the average volume of sample was smallest. Microfluidics-FISH proved to be reliable and cost-effective rapid testing method of common aneuploidies. Recognizing, however, limitations of methods based on FISH it cannot replace conventional karyotyping and be the sole method of diagnosis. Key words: Microfluidics-FISH, rapid fish, rapid aneuploidy testing, amniocentesis, prenatal diagnosis
\end{abstract}

Ginekologia Polska 2017; 88, 12: 670-673

\section{INTRODUCTION}

The aim of prenatal diagnosis is to detect congenital abnormalities of the fetus. Increasing number of defects can be treated in uterus or corrected after birth but a distinct amount of them is irreversible or even lethal. In those cases prenatal diagnosis allows parents to make an informed decision about course of the pregnancy as well health care professionals to optimize ante- and postnatal care. The most frequently identified chromosome abnormality in humans is aneuploidy (trisomy or monosomy), which occurs in at least $5 \%$ of all clinically recognized pregnancies [1]. Trisomy for 13, 18 and 21 chromosome and X, Y chromosome aneuploidies are the most common prenatally diagnosed aberrations [2] and are responsible for more than $90 \%$ of live- born chromosomal abnormalities [3].
With application of modern molecular techniques such as: FISH, MLPA, QF-PCR, BoBs and aCGH allowing for much faster aneuploidy detection, new strategies have evolved. FISH, introduced to prenatal diagnosis in 1992 has proved to be effective and reliable method of detection on uncultured samples and considerably reduced reporting time [4-6]. Most centers applying this technique use commercially validated probe sets, which are expensive, and the protocols that are labor- intensive and time-consuming [7].

Microfluidics-FISH is a novel approach to prenatal diagnosis in which a special device, MicroFIND ${ }^{\circledR}$, is used. This IVD certified microchip allows to combine different steps of FISH into integral process, shortening the procedure so that results are available within 24 hours of amniocentesis. With reduced price of single test and shorter turnaround time 
Microfluidics-FISH seems competitive to other available methods.

\section{OBJECTIVES}

In this study we evaluate the use of Microfluidics-FISH in prenatal diagnosis comparing it to other methods commonly used in that field-classical karyotype and Rapid FISH.

\section{MATERIAL AND METHODS}

Samples of amniotic fluid were obtained from 52 pregnant women between $15+1$ and $28+5$ weeks of pregnancy basing on last menstrual period (LMP) or biparietal diameter (BPD) evaluated in ultrasound scan. The amniocenteses were performed in a consecutive series in one prenatal center due to standard indications: increased risk of common chromosomal aberrations in prenatal screening, advanced maternal age, abnormalities in ultrasound scan, suspicion of congenital defects or chromosomal aberration in preceding pregnancy, risk of monogenic disorder and extreme parental stress. Prior to that all women were counseled by medical geneticist, confirmed they understood disclosed information and gave their written informed consent. Each obtained sample of amniotic fluid (19-22 mL) was distributed for: karyotyping (10-14 mL), Rapid FISH (4-6 mL) and Microfluidics-FISH (1-4 mL), which were performed according to standard protocols adopted in our laboratory.

In rare cases of Rapid FISH or Microfluidics-FISH failure the protocol was extended. Samples were reevaluated after repeating dehydration process and following steps with increased amount of probes and hybridization time.
The comparison of results obtained with each applied technique was performed and statistical differences were measured with chi square test.

Sample collection for research was approved by Bioethics Committee of Pomeranian Medical University in Szczecin, Poland.

\section{RESULTS}

Of the 52 analyzed amniotic samples, chromosomal aberrations have been found in 10 of them, what comprises almost 20\%. The most recurrent abnormality was trisomy for 21 chromosome, which occurred in 7 fetuses. All cases were classical trisomy and were also detected by both FISH methods. Other aberrations included single case of: mosaic trisomy for 18 chromosome (dominating trisomic cell line was identified in comparative percentage in all techniques), triploid (evident in all methods) and small supernumerary marker chromosome (sSMC) that was observed only in classical karyotyping but was not identified by Rapid FISH and Microfluidics-FISH. In the group in which abnormality was detected the accordance rate of both FISH techniques and classical cytogenetic analyses was $98 \%$ and the difference was not statistically significant.

In remaining group of 42 cases, which consisted of 24 female and 18 male fetuses no other aberration was found as their results were assessed as normal in all techniques. An overview of anomalies found in the classical karyotype, juxtaposed to the results of both FISH methods, is shown in Table 1.

Standard protocol allowed to obtain indicative results of classical karyotype in all patients. Regarding Rapid FISH and

Table 1. The overview of anomalies detected by classical karyotype, compared to results of Rapid FISH and Microfluidics-FISH

\begin{tabular}{|c|c|c|c|c|c|c|c|c|}
\hline Result & Sex & $\begin{array}{c}\text { Classical } \\
\text { karyotype }\end{array}$ & Rapid FISH & \multicolumn{2}{|c|}{$\begin{array}{c}\text { Accordant } \\
\text { to karyotype \% }\end{array}$} & Microfluidics-FISH & \multicolumn{2}{|c|}{$\begin{array}{c}\text { Accordant } \\
\text { to karyotype \% }\end{array}$} \\
\hline \multirow{3}{*}{ Normal } & Female & 24 & 24 & Yes & $100 \%$ & 24 & Yes & $100 \%$ \\
\hline & Male & 18 & 18 & Yes & $100 \%$ & 18 & Yes & $100 \%$ \\
\hline & Total & 42 & 42 & Yes & $100 \%$ & 42 & Yes & $100 \%$ \\
\hline \multicolumn{9}{|l|}{ Abnormal } \\
\hline \multirow{2}{*}{ Trisomy 21} & Female & 4 & 4 & Yes & $100 \%$ & 4 & Yes & $100 \%$ \\
\hline & Male & 3 & 3 & Yes & $100 \%$ & 3 & Yes & $100 \%$ \\
\hline Triploidy & Female & 1 & 1 & Yes & $100 \%$ & 1 & Yes & $100 \%$ \\
\hline Mosaic trisomy 18 & Female & 1 & 1 & Yes & $100 \%$ & 1 & Yes & $100 \%$ \\
\hline SSMC & Male & 1 & 0 & No & $0 \%$ & 0 & No & $0 \%$ \\
\hline \multirow{3}{*}{ All abnormal } & Female & 6 & 6 & Yes & $100 \%$ & 6 & Yes & $100 \%$ \\
\hline & Male & 4 & 3 & No & $75 \%(3 / 4)$ & 3 & No & $75 \%(3 / 4)$ \\
\hline & Total & 10 & 9 & No & $90 \%(9 / 10)$ & 9 & No & $90 \%(9 / 10)$ \\
\hline All & All & 52 & 52 & No & $98 \%(51 / 52)$ & 52 & No & $98 \%(51 / 52)$ \\
\hline
\end{tabular}


Table 2. Cost and time analysis

\begin{tabular}{|c|c|c|c|c|c|c|c|c|}
\hline & \multicolumn{5}{|c|}{ TIME } & \multicolumn{3}{|c|}{$\operatorname{cosT}$} \\
\hline & \multicolumn{2}{|c|}{ SP* } & \multicolumn{2}{|c|}{ EP* } & \multirow{2}{*}{ Single assay } & \multirow{2}{*}{ SP* } & \multirow{2}{*}{ EP* } & \multirow{2}{*}{$\begin{array}{c}\text { Single } \\
\text { assay }\end{array}$} \\
\hline & Range & Mean & Range & Mean & & & & \\
\hline Classical karyotype & $10-20$ days & 17 days & - & - & 17 days & 1 & - & 1 \\
\hline Rapid FISH & $40-48 \mathrm{~h}$ & $43 \mathrm{~h}$ & $+24 h$ & $67 \mathrm{~h}$ & $-44 h$ & 0.78 & 1.2 & 0.8 \\
\hline Microfluidics-FISH & $8-24 h$ & $20 \mathrm{~h}$ & $+24 \mathrm{~h}$ & $34 \mathrm{~h}$ & $-21.5 h$ & 0.35 & 0.55 & 0.37 \\
\hline
\end{tabular}

*SP — standard protocol; EP — extended protocol

Microfluidics-FISH method it was $96 \%$ and $90 \%$ of samples, respectively. In order to achieve diagnostic result in 2 cases of Rapid FISH and 5 cases of Microfluidics-FISH extended protocol had to be applied. The need for reassessment was attributed to poor quality of samples related to their advanced gestational age (20-29 weeks). No such problems were encountered in samples from 15-19 week of pregnancy.

The turnaround time was shortest for Microfluidics-FISH and ranged from 9 to 24 hours, depending on the quality of sample and other factors such as time of admission to the lab. The need to apply extended protocol doubled the time required to achieve diagnostic result for Microfluidics-FISH, as shown in Table 2.

The determined cost was lowest for Microfluidics-FISH. It was nearly three and two times reduced comparing to classical karyotype and Rapid FISH, respectively. The need for extended protocol, which arose in almost $10 \%$ of cases in Microfluidics-FISH nearly, doubled the cost of a single assay. The cost analysis is also given in Table 2.

\section{DISCUSSION}

In our study Microfluidics-FISH proved to be an accurate and cost-effective rapid testing method of common aneuploidies, what is also consistent with results published by Yo et al. [8]. Chromosomal aberrations, existing in $20 \%$ of investigated samples, have been identified correctly by all methods in all but one case, in which fetus was diagnosed with SSMC. SSMC, which can only be seen in standard karyotyping, occurs at a rate of $0.65-1.5 / 1000$ in prenatal diagnosis [9]. In our patient further molecular analysis revealed it to be of 22 chromosome origin. As available kit of FISH probes is focused on 13, 18, 21, $\mathrm{X}$ and $\mathrm{Y}$ chromosome aneuploidies, this abnormality could not be detected and presents limitation of methods based on FISH. Almost 30\% of chromosomal aberrations might not be detected by FISH based methods in prenatal diagnosis, concluded Evans et al. in their study which was an international, collaborative assessment of 146000 prenatal cytogenetic results [10]. However, the question arises about their clinical relevance as balanced translocations were also counted. The prevalence of clinically significant aberrations that are not detected by rapid testing has been estimated at around $0.1 \%$ by Ogilvie et al. [7].

The most recurrent abnormality, trisomy for 21 chromosome was recognized appropriately in all samples and no false positive or negative results have been encountered in both FISH methods. Additionally, there was $100 \%$ accordance between FISH detected trisomy and ultrasound anomalies.

The most distinguishing feature of the group in which reevaluation with extended protocol was required, was their advanced gestational age (20-29 weeks) what relates to poor quality sample: reduced number of viable amniotic cells, excessive amount of cell debris and residual cytoplasm. The same problem was encountered in aforementioned study by Yo et al. [8]. In such cases, a higher concentration of pellet was needed and loading process had to be repeated. Altogether, it considerably increased cost and reporting time and linked with information about the need to reexamine deepened parental stress.

The volume of sample needed to perform test was smallest for Microfluidics-FISH what was beneficial in cases of oligohydroamnios and when amniocentesis was technically difficult to perform. As only 1, $5 \mu$ l of pellet was required for a single analysis, in the event of its failure the remaining specimen could be tested.

Comparing Microfluidics-FISH to the evaluated methods, it was most affordable as its estimated cost comprised one third of the standard karyotype and half of the Rapid FISH. Taking into consideration other low-cost, molecular methods available on market, such as MLPA or QF-PCR the price of a single assay is still more competitive.

The turnaround time was also shortest for Microfluidics-FISH and ranged from 9 to 24 hours. Under favorable conditions (sample admission in the morning, early pregnancy) the result was given within the same day of amniocentesis not compromising on its quality.

In light of these facts, the targeted group of Microfluidics-FISH test seems to be patients, in which amniocentesis was performed between 15-19 week of gestation due to very specific indications, increased risk of Down syndrome being the main one. As it is still leading reason of referral, that group is dominant in prenatal centers. 
The role of FISH based method as the only applied test in prenatal diagnosis has not been clearly defined and varying strategies maybe be adopted in different facilities. Yet affordable price and accelerated turnaround time of Microfluidics-FISH make it a valuable additional or exclusive test that allows to alleviate parental anxiety and advance further care and pregnancy management.

\section{Acknowledgements}

This research was partially supported by Pomeranian Medical University (Young Scientist subsidy No.158-110/14).

\section{REFERENCES}

1. HassoldT, Hunt P.To err (meiotically) is human: the genesis of human aneuploidy. Nat Rev Genet. 2001; 2(4): 280-291, doi: 10.1038/35066065, indexed in Pubmed: 11283700.

2. Divane A, Carter NP, Spathas DH, et al. Rapid prenatal diagnosis of aneuploidy from uncultured amniotic fluid cells using five-colour fluorescence in situ hybridization. Prenat Diagn. 1994; 14(11): 1061-1069, doi: 10.1002/pd.1970141109, indexed in Pubmed: 7877954

3. Philip J, Bryndorf T, Christensen B. Prenatal aneuploidy detection in interphase cells by fluorescence in situ hybridization (FISH). Prenat Diagn. 1994; 14(13): 1203-1215, indexed in Pubmed: 7617567.
4. Klinger K, Landes G, Shook D, et al. Rapid detection of chromosome aneuploidies in uncultured amniocytes by using fluorescence in situ hybridization (FISH). Am J Hum Genet. 1992; 51(1): 55-65, indexed in Pubmed: 1609805.

5. Tepperberg J, Pettenati MJ, Rao PN, et al. Prenatal diagnosis using interphase fluorescence in situ hybridization (FISH): 2-year multi-center retrospective study and review of the literature. Prenat Diagn. 2001; 21(4): 293-301, doi: 10.1002/pd.57, indexed in Pubmed: 11288120.

6. Witters I, Devriendt K, Legius $\mathrm{E}$, et al. Rapid prenatal diagnosis of trisomy 21 in 5049 consecutive uncultured amniotic fluid samples by fluorescence in situ hybridisation (FISH). Prenat Diagn. 2002; 22(1): 29-33, doi: 10.1002/pd.225, indexed in Pubmed: 11810646.

7. Ogilvie CM, Lashwood A, Chitty L, et al. The future of prenatal diagnosis: rapid testing or full karyotype? An audit of chromosome abnormalities and pregnancy outcomes for women referred for Down's Syndrome testing. BJOG. 2005; 112(10): 1369-1375, doi: 10.1111/j.1471-0528.2005.00695.x, indexed in Pubmed: 16167939.

8. Ho SSY, Chua C, Gole L, et al. Same-day prenatal diagnosis of common chromosomal aneuploidies using microfluidics-fluorescence in situ hybridization. Prenat Diagn. 2012; 32(4): 321-328, doi: 10.1002/pd.2946, indexed in Pubmed: 22467162.

9. Graf MD, Christ L, Mascarello JT, et al. Redefining the risks of prenatally ascertained supernumerary marker chromosomes: a collaborative study. J Med Genet. 2006; 43(8): 660-664, doi: 10.1136/jmg.2005.037887, indexed in Pubmed: 16882740.

10. Evans MI, Henry GP, Miller WA, et al. International, collaborative assessment of 146,000 prenatal karyotypes: expected limitations if only chromosome-specific probes and fluorescent in-situ hybridization are used. Hum Reprod. 1999; 14(5): 1213-1216, doi: 10.1093/humrep/14.5.1213, indexed in Pubmed: 10325263. 